\title{
Trends in isolated coronary artery bypass grafting: An analysis of the Society of Thoracic Surgeons adult cardiac surgery database
}

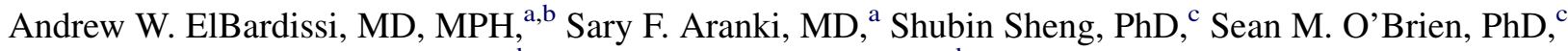 \\ Caprice C. Greenberg, MD, MPH, ${ }^{b}$ and James S. Gammie, MD $^{\mathrm{d}}$
}

\begin{abstract}
Objective: Coronary artery bypass grafting $(\mathrm{CABG})$ is the operation most commonly performed by cardiac surgeons. There are few contemporary data examining evolving patient characteristics and surgical outcomes of isolated CABG. We used the Society of Thoracic Surgeons adult cardiac surgery database to characterize trends in patient characteristics and outcomes after CABG over the past decade.
\end{abstract}

\begin{abstract}
Methods: From 2000 to 2009, 1,497,254 patients underwent isolated primary CABG at Society of Thoracic Surgeons participating institutions. Demographics, operative characteristics, and postoperative outcomes were assessed, and risk-adjusted outcomes were calculated.

Results: Compared with the year 2000, patients undergoing isolated primary CABG in 2009 were more likely to have diabetes mellitus ( $33 \%$ vs $40 \%$ ) and hypertension ( $71 \%$ vs $85 \%)$. There were clinically insignificant differences in age, gender, and body surface area. Between 2000 and 2009, there has been a $6.3 \%$ and $19.5 \%$ increase in the preoperative use of aspirin and beta-blockers, respectively. Between 2004 and 2009, there was a $7.8 \%$ increase in the use of angiotension-converting enzyme inhibitors preoperatively. Furthermore, between 2005 and 2009 there was a $3.8 \%$ increase in the use of statins preoperatively. The median number of distal anastomoses performed was unchanged between 2000 and 2009 ( 3 ; interquartile range, 2-4). There was a significant increase in the use of the internal thoracic artery ( $88 \%$ in $2000 \mathrm{vs} 95 \%$ in 2009). The predicted mortality rates of $2.3 \%$ were consistent between 2000 and 2009 . The observed mortality rate over this period declined from $2.4 \%$ in 2000 to $1.9 \%$ in 2009 representing a relative risk reduction of $24.4 \%$. The incidence of postoperative stroke decreased significantly from $1.6 \%$ to $1.2 \%$, representing a risk reduction of $26.4 \%$. There was also a $9.2 \%$ relative reduction in the risk of reoperation for bleeding and a $32.9 \%$ relative risk reduction in the incidence of sternal wound infection.
\end{abstract}

Conclusions: Over the past decade, the risk profile of patients undergoing CABG has changed, with fewer smokers, more diabetic patients, and better medical therapy characterizing patients referred for surgical coronary revascularization. The left internal thoracic artery is nearly universally used and outcomes have improved substantially, with a significant decline in postoperative mortality and morbidity. (J Thorac Cardiovasc Surg 2012;143:273-81)

Although coronary artery disease (CAD) continues to be the leading cause of morbidity and mortality in the developed world, epidemiologic evidence suggests that there has

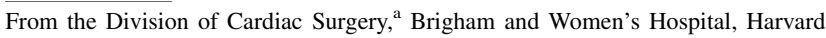
Medical School, Boston, Mass; Center for Surgery and Public Health, ${ }^{\mathrm{b}}$ Brigham and Women's Hospital, Boston, Mass; Outcomes Research and Assessment Group, ${ }^{\mathrm{c}}$ Duke Clinical Research Institute, Durham, NC; and Division of Cardiac Surgery, ${ }^{\mathrm{d}}$ University of Maryland School of Medicine, Baltimore, Md.

Dr ElBardissi is supported by the Agency for Health Care Research Quality National Research Service Award Grant 1F32HS019190 and the Arthur Tracy Cabot Fellowship at Brigham and Women's Hospital and the Center for Surgery and Public Health. The Society of Thoracic Surgeons through the Adult National Cardiac Database and the Duke Clinical Research Institute supported this work.

Disclosures: Authors have nothing to disclose with regard to commercial support.

Read at the 91st Annual Meeting of The American Association for Thoracic Surgery, Philadelphia, Pennsylvania, May 7-11, 2011.

Received for publication May 8, 2011; revisions received Sept 23, 2011; accepted for publication Oct 20, 2011.

Address for reprints: Andrew W. ElBardissi, MD, MPH, Division of Cardiac Surgery, Brigham and Woman's Hospital, 75 Francis St, Boston, MA 02115 (E-mail: aelbardissi@partners.org).

0022-5223/\$36.00

Copyright (c) 2012 by The American Association for Thoracic Surgery

doi:10.1016/j.jtcvs.2011.10.029 been a dramatic reduction in deaths attributable to coronary heart disease in recent decades. ${ }^{1}$ Reasons for this decline include a reduction in risk factors, such as smoking, hypercholesterolemia, and hypertension, and widespread use of medical therapy, including antihypertensives, betablockers, angiotensin-converting enzyme inhibitors, and statins. $^{2}$ In addition, the introduction and widespread use of surgical and interventional revascularization techniques have further led to a reduction in mortality attributable to coronary heart disease. ${ }^{3}$

Since its introduction in 1962, coronary artery bypass grafting $(\mathrm{CABG})$ has continued to be the gold standard for revascularization of $\mathrm{CAD}$, particularly in high-risk patients with multivessel disease. ${ }^{4}$ Evidence from multiple studies suggests that $\mathrm{CABG}$ prolongs survival especially in complex patients with diabetes, ${ }^{3}$ those aged 65 years or more,${ }^{3,5}$ those with left main stem or triple-vessel disease,${ }^{6}$ and those with impaired left ventricular function. ${ }^{6}$ The Synergy Between PCI With TAXUS and Cardiac Surgery (SYNTAX) clinical trial $^{4}$ revealed that rates of major adverse cardiac or 


$$
\begin{aligned}
& \text { Abbreviations and Acronyms } \\
& \begin{aligned}
\text { CABG } & =\text { coronary artery bypass grafting } \\
\text { CAD } & =\text { coronary artery disease } \\
\text { IABP } & =\text { intraaortic balloon pump } \\
\text { ITA } & =\text { internal thoracic artery } \\
\text { PCI } & =\text { percutaneous coronary intervention } \\
\text { RRR } & =\text { relative risk reduction } \\
\text { STS } & =\text { Society of Thoracic Surgeons } \\
\text { SYNTAX } & =\text { Synergy Between PCI With TAXUS } \\
& \text { and Cardiac Surgery }
\end{aligned}
\end{aligned}
$$

cerebrovascular events at 12 months were significantly lower in patients with 3-vessel or left main disease who underwent $\mathrm{CABG}$ compared with percutaneous coronary intervention (PCI). Despite significant developments in PCI, CABG remains the most commonly used treatment option for patients with complex CAD and high-risk patients. ${ }^{7}$

Despite the known and dramatic effectiveness of CABG, the use of PCI as a method of revascularization has grown at a significant rate since its introduction in $1977^{\circ}$ and has contributed to a decline in the number of CABGs performed annually. ${ }^{9}$ Given these trends in the incidence of CAD, medical treatment effectiveness, and alternative interventional CAD treatment strategies, a number of important clinical questions arise: (1) Are patients undergoing CABG today characteristically different from patients undergoing $C A B G$ in the past? (2) Have risk-adjusted outcomes improved? (3) Has the choice of conduits used in CABG evolved?

The purpose of this study is to define how the characteristics of patients undergoing isolated CABG has changed during the period of 2000 to 2009 , to further characterize improvements in risk-adjusted clinical outcomes, and to assess how surgical decision-making has changed in the preceding decade with regard to the number and type of conduits used.

\section{MATERIALS AND METHODS Study Population}

The study population included patients aged 18 years or more who underwent first-time isolated CABG at participating hospitals of the Society of Thoracic Surgeons (STS) between January 1, 2000, and December 31, 2009. Patients undergoing repeat CABG and patients with missing data on age, gender, or acuity status were excluded.

\section{Data Elements}

Data were collected using 4 versions of the STS data collection as follows: v2.35 (years 2000-2002), v2.41 (years 2002-2004), v2.52 (years 2004-2007), and v2.61 (years 2008-2009). Note that 2 versions were in use simultaneously during parts of 2002 and 2004. Definitions for the various versions of the STS database can be found at http://www.sts.org/ quality-research-patient-safety/national-database/database-managers/adultcardiac-surgery-database/d. Definitions were reviewed by the authors to determine whether study variables had comparable definitions over time.
Variables that changed substantially were excluded from analysis or analyzed within shorter time periods during which the definitions were consistent. Variables that were analyzed over shorter time periods are labeled in the appropriate tables. Variables that had minor definitional changes and were still used for analysis are shown below with the definitional changes that occurred:

\section{Preoperative Variable Definitional Changes}

- History of cerebrovascular accident: Symptoms persisting for more than 24 hours after initial onset in versions 2.35 and 2.61, and symptoms persisting for more than 72 hours after initial onset in versions 2.41 and 2.52 .

- Preoperative arrhythmia: Preoperative arrhythmia present within 2 weeks of the procedure by clinical documentation of any one of the following: atrial fibrillation/flutter requiring treatment, heart block, or sustained ventricular tachycardia or ventricular fibrillation requiring cardioversion or intravenous amiodarone in versions 2.35 and 2.41 versus history of preoperative arrhythmia (sustained ventricular tachycardia, ventricular fibrillation, atrial fibrillation, atrial flutter, third-degree heart block) that has been clinically documented or treated with any of the following treatment modalities: (1) ablation therapy, (2) automatic implantable cardioverter-defibrillator, (3) pacemaker, (4) pharmacologic treatment, or (5) electrocardioversion in versions 2.52 and 2.61.

- Procedure status (emergency): The patient's clinical status includes any of the following: (a) ischemic dysfunction (any of the following): (1) ongoing ischemia including rest angina despite maximal medical therapy (medical or intraaortic balloon pump [IABP]), (2) acute evolving myocardial infarction within 24 hours before surgery, or (3) pulmonary edema requiring intubation. (b) Mechanical dysfunction (either of the following): (1) shock with circulatory support or (2) shock without circulatory support in versions $2.35,2.41$, and 2.52 versus patients requiring emergency operations will have ongoing, refractory (difficult, complicated, or unmanageable), unrelenting cardiac compromise, with or without hemodynamic instability, and not responsive to any form of therapy except cardiac surgery. An emergency operation is one in which there should be no delay in providing operative intervention in version 2.61.

- Preoperative medications (aspirin, beta-blockers, steroids): Version 2.35 did not indicate time period before surgery, whereas $2.41,2.52$, and 2.61 had the following time intervals: acetylsalicylic acid ( 5 days before surgery), beta-blockers ( 24 hours before surgery), angiotensin-converting enzyme inhibitors ( 24 hours before surgery), and steroids ( 24 hours before surgery).

- Preoperative medications (statins): Not included in version 2.35; included in versions $2.41,2.52$, and 2.61 with consistent definitions.

\section{Outcome Variable Definitional Changes}

- Deep sternal wound infection: Sternal infection/superficial (skin, subcutaneous tissue) must have one of the following conditions: (1) wound opened with excision of tissue (incision and drainage), (2) positive culture, or (3) treatment with antibiotics in version 2.35 versus deep sternal infection within 30 days postoperatively, involving muscle, bone, or mediastinum requiring operative intervention. Must have all of the following conditions: (1) wound opened with excision of tissue (incision and drainage) or reexploration of mediastinum, (2) positive culture, and (3) treatment with antibiotics. In versions $2.41,2.52$, and 2.61.

- Stroke: A central neurologic deficit persisting for more than 72 hours (versions $2.35,2.41$, and 2.52) versus postoperative stroke that did not resolve in 24 hours (version 2.61).

\section{Definition of Study End Points}

- Operative mortality defined as death during the same hospitalization as surgery or after discharge but within 30 days of surgery. 
- Stroke defined as a central neurologic deficit persisting postoperatively for more than 24 hours (note that in 2009 definition changed from $>24$ hours to $>72$ hours).

The secondary end points of interest included the following:

- Reoperation for bleeding defined as an operative reintervention for bleeding or tamponade.

- Renal failure defined as an increase of serum creatinine to greater than 2.0 and 2 times most recent preoperative creatinine level or a new requirement for dialysis postoperatively.

- Deep sternal infection, defined as a deep sternal infection involving muscle, bone, or mediastinum with the following requirements: wound opened with excision of tissue (incision and drainage), positive culture, or treatment with antibiotics.

- Postoperative length of stay.

- New-onset postoperative atrial fibrillation.

\section{Statistical Analysis}

Patient characteristics and outcomes were summarized as frequency and percentage, or as median and interquartile range (25th-75th percentile). Differences in the prevalence of risk factors in 2000 versus 2009 were assessed with stratified Mantel-Haenszel chi-square tests or Wilcoxon ranksum tests, with stratification by STS participant identity. For comparing outcomes across 2 time points, the percentage relative risk reduction (RRR) was calculated as $100 \times\left(\mathrm{P}_{2}-\mathrm{P}_{1}\right) / \mathrm{P}_{1}$, where $\mathrm{P}_{1}$ and $\mathrm{P}_{2}$ denote the percentage of patients experiencing the end point in the earlier and later time periods, respectively. Confidence intervals for the RRR were calculated by approximating the number of deaths in each time period as a Poisson distribution.

To create a patient-level summary measure of case severity, we used logistic regression modeling to estimate the probability of mortality for each patient in the study sample. Covariates for predicting mortality included age, gender, body surface area, ejection fraction, preoperative dialysis, preoperative atrial fibrillation, cardiogenic shock, immunosuppressive treatment, PCI less than 6 hours, preoperative intra-aortic balloon pump or inotrope, peripheral vascular disease, unstable angina (no myocardial infarction $<7$ days), left main disease, cerebrovascular disease (CVD)/cerebrovascular accident, diabetes, number of disease vessels, myocardial infarction, operative urgency status, and congestive heart failure/New York Heart Association class. Covariates were selected on the basis of having a previously documented association with mortality, ${ }^{10}$ a low rate of missing data, and consistent definitions across the study period. Parameters of the logistic model were estimated using generalized estimating equations with an exchangeable correlation structure to account for withincenter clustering. The patient's estimated probability of death was regarded as a simple summary measure that combines several individual risk factors into a single number. Trends in case severity over time were assessed by calculating the average estimated probability of death among all patients in the same calendar year to obtain the year-specific predicted mortality prevalence rate. Analogous models and measures were also created for the 6 nonfatal end points.

Trends in short-term mortality and morbidity outcomes over time were assessed by calculating unadjusted and risk-adjusted prevalence rates for each study end point by calendar year. The unadjusted prevalence rate was defined as the proportion of patients in a given calendar year who had the end point of interest. The risk-adjusted prevalence rate for a given end point and calendar year was calculated by dividing the year-specific unadjusted prevalence rate of the end point (defined above) by the yearspecific predicted prevalence rate of the end point (defined in the previous paragraph) and then multiplying by the overall proportion of patients who had the end point during the entire study period. The calculation of riskadjusted RRRs and associated confidence intervals was similar to the unadjusted versions described above. Given the large sample size, $P$ values will not be reported because practically every difference is statistically significant, however, not necessarily clinically significant.

STS data were highly complete with most study variables missing less than $1 \%$ in each calendar year. Missing data rates were highest in 2000 and generally lower in each successive calendar year. Covariates with the most missing data had the following missing data rates for calendar years 2000 and 2009: ejection fraction $(7.8 \%, 3.5 \%)$, use of ITA $(4.6 \%, 0.0 \%)$, and off-pump procedure $(3.3 \%, 0.0 \%)$. Remaining variables had less than $3 \%$ missing in each calendar year or are explicitly stated in data tables. For each variable, summary measures are based on the subset of patients without missing data.

\section{RESULTS \\ Patient Population}

From 2000 to 2009, 2,473,313 patients underwent cardiac surgery at 1073 institutions participating in the STS Adult Cardiac Surgery Database (Figure 1). After exclusion of patients undergoing non-CABG procedures $(28 \%)$, patients undergoing concomitant operations $(11.7 \%)$, patients undergoing reoperative CABGs $(4.6 \%)$, and patients with missing age or gender data $(0.1 \%), 1,497,254$ patients make up the study base for this analysis.

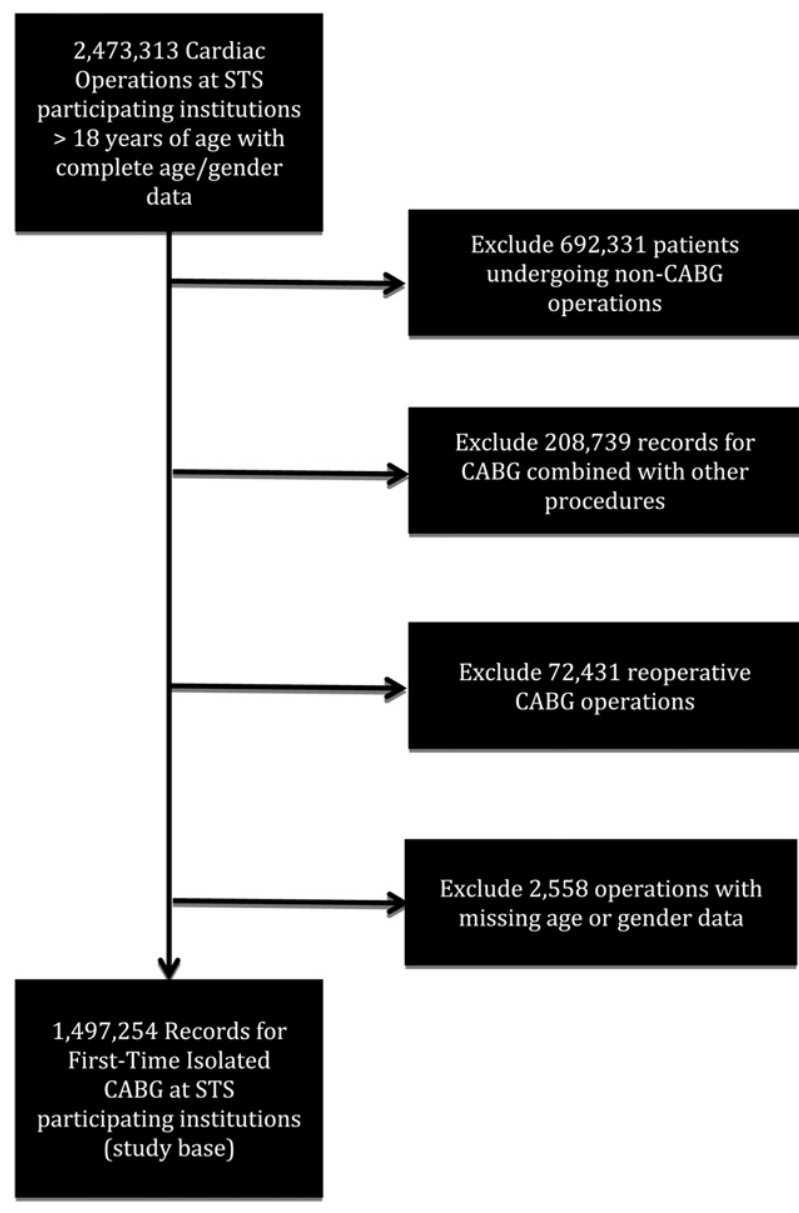

FIGURE 1. Enrollment and exclusion criteria. $C A B G$, Coronary artery bypass grafting; STS, Society of Thoracic Surgeons. 


\section{Characteristics of Patients Undergoing Coronary Artery Bypass Grafting}

As shown in Table 1, between 2000 and 2009 there has been a modest increase in diabetes mellitus and a significant increase in hypertension. There were minor differences in age and body surface area over time. Use of statins, aspirin, beta-blockers, and angiotensin-converting enzyme inhibitors increased by $3.8 \%, 6.3 \%, 19.5 \%$, and $7.8 \%$, over their respective periods of data collection (see Table 1 footnote). Further, there was a $37 \%$ increase the number of patients who underwent PCI with or without stenting before undergoing CABG (19\% vs $26 \%)$.

Table 2 highlights the hemodynamic and catheterization characteristics of patients undergoing primary $\mathrm{CABG}$. The percent of patients with left main disease increased from $22.6 \%$ in 2000 to $31.6 \%$ in 2009 . The median ejection fraction increased from $50 \%$ in 2000 to $55 \%$ in 2009 , whereas the incidence of 3-vessel disease increased marginally.

\section{Operative Characteristics}

As shown in Table 3, there has been an increase in the use of the left internal thoracic artery (ITA) for coronary revascularization, increasing from $87.7 \%$ in 2000 to $94.7 \%$ in 2009 . Use of the isolated right ITA for primary CABGs remained constant at less than $0.6 \%$ throughout the study, whereas use of bilateral ITAs increased slightly from $3.5 \%$ in 1999 to $4.1 \%$ in 2009 . The radial artery also had varying rates of adoption since 2000 when it was used in $10.8 \%$ of all primary CABGs. Use of the radial artery peaked at $12.3 \%$ in 2002 and subsequently declined to $5.5 \%$ in 2009.

As a proportion of total procedures (Table 3), elective CABG is being performed less in 2009 than in 2000 (57.6\% elective in 2000 vs $41.3 \%$ elective in 2009 ). There was a concomitant increase in the number of urgent cases $(38.0 \%$ vs $54.1 \%)$, although emergency and emergencysalvage cases remained constant throughout the study. Finally, although the timing of IABP implantation seems to have changed over time (more patients with IABP preoperatively and fewer with IABP intraoperatively and postoperatively), the overall proportion of patients receiving an IABP has remained constant at approximately $10 \%$ (Table 3). Perfusion and crossclamp times remained relatively constant in this study, although the proportion of

TABLE 1. Preoperative demographics of primary coronary artery bypass grafting between 2000 and 2009

\begin{tabular}{|c|c|c|}
\hline & 2000 & 2009 \\
\hline \multicolumn{3}{|l|}{ Demographics } \\
\hline Age & $65(57-73)$ & $65(57-73)$ \\
\hline Male gender & $70.6 \%$ & $72.9 \%$ \\
\hline White race & $92.0 \%$ & $85.0 \%$ \\
\hline Weight (kg) & $84(73-95)$ & $86(75-100)$ \\
\hline BSA & $1.97(1.81-2.12)$ & $2.00(1.84-2.16)$ \\
\hline Diabetes & $33.4 \%$ & $40.4 \%$ \\
\hline Hypercholesterolemia & $60.4 \%$ & $83.8 \%$ \\
\hline Renal failure (preoperative dialysis) & $1.2 \%$ & $2.5 \%$ \\
\hline COPD & $16.9 \%$ & $22.7 \%$ \\
\hline Hypertension & $70.9 \%$ & $85.0 \%$ \\
\hline Peripheral vascular disease & $14.9 \%$ & $14.7 \%$ \\
\hline No $\mathrm{CHF}$ & $87.0 \%$ & $84.1 \%$ \\
\hline CHF and NYHA class IV & $5.0 \%$ & $4.0 \%$ \\
\hline CHF and no NYHA class IV & $7.9 \%$ & $11.9 \%$ \\
\hline Previous CVA & $5.9 \%$ & $6.7 \%$ \\
\hline CVD without previous CVA & $5.9 \%$ & $7.4 \%$ \\
\hline Preoperative arrhythmia & $10.0 \%$ & $8.6 \%$ \\
\hline \multicolumn{3}{|l|}{ Previous interventions } \\
\hline Preoperative PCI (with or without stenting) $<6 \mathrm{~h}$ & $0.82 \%$ & $0.94 \%$ \\
\hline Any prior PCI (with or without stenting) & $19.1 \%$ & $25.6 \%$ \\
\hline Previous valve surgery & $0.2 \%$ & $0.2 \%$ \\
\hline Previous other cardiac surgery & $1.8 \%$ & $1.4 \%$ \\
\hline \multicolumn{3}{|l|}{ Preoperative medications } \\
\hline Aspirin & $73.1 \%$ & $79.4 \%$ \\
\hline Beta blockers & $61.9 \%$ & $81.4 \%$ \\
\hline ACE inhibitors* & $38.4 \%$ & $46.2 \%$ \\
\hline Statin $\dagger$ & $60.6 \%$ & $64.4 \%$ \\
\hline
\end{tabular}

In year 2000, ACE inhibitor and statin missing data exceeded 3\%.ACE, Angiotensin-converting enzyme; $B S A$, body surface area; $C H F$, congestive heart failure; $C O P D$, chronic obstructive pulmonary disease; CVA, cerebrovascular accident; NYHA, New York Heart Association; PCI, percutaneous coronary intervention. *Data are from 2004, not 2000. $\dagger$ Data are from 2005, not 2000. 
TABLE 2. Coronary lesions and ejection fraction

\begin{tabular}{lcc}
\hline \multicolumn{1}{c}{ Hemodynamics } & $\mathbf{2 0 0 0}$ & $\mathbf{2 0 0 9}$ \\
\hline Demographics & & \\
Left main disease $>50 \%$ & $22.6 \%$ & $31.6 \%$ \\
Number of diseased vessels & & \\
1 & $4.7 \%$ & $4.5 \%$ \\
2 & $21.1 \%$ & $20.5 \%$ \\
3 & $73.8 \%$ & $74.7 \%$ \\
Ejection fraction & $50(40-60)$ & $55(45-60)$ \\
\hline
\end{tabular}

cases performed off-pump increased from $14.5 \%$ in 2000 to $21.1 \%$ in 2009.

\section{Outcomes in Total Cohort}

As shown in Figure 2, $A$ and Table 4, the change in patient demographic and preoperative risk resulted in a relatively constant predicted mortality rate throughout the duration of this study. There was a $24.4 \%$ and $26.4 \%$ reduction in the unadjusted observed operative mortality $(2.4 \%$ vs $1.9 \%)$ and stroke rates $(1.6 \%$ vs $1.2 \%)$, respectively, during the course of the study period (Table 4). There was also a $9.2 \%$ RRR in the incidence of reoperation for bleeding $(2.5 \%$ vs $2.2 \%$, Table 4$)$ and a $32.9 \%$ relative reduction in the incidence of sternal wound infection $(0.55 \%$ vs $0.37 \%$, Table 4 ). There was a $5.5 \%$ relative increase in the incidence of new-onset renal failure between 2000 and 2009 and a $6.5 \%$ relative increase in the incidence of new postoperative atrial fibrillation (Table 4). Median length of stay was 5 days (interquartile range, 4-7) and did not change throughout the course of the study.

TABLE 3. Operative characteristics

\begin{tabular}{lcc}
\hline Operative characteristics & $\mathbf{2 0 0 0}$ & $\mathbf{2 0 0 9}$ \\
\hline Use of internal thoracic & $87.7 \%$ & $94.7 \%$ \\
Off-pump procedure & $14.5 \%$ & $21.1 \%$ \\
Procedure status & & \\
Elective & $57.6 \%$ & $41.3 \%$ \\
Urgent & $38.0 \%$ & $54.1 \%$ \\
Emergency & $4.2 \%$ & $4.4 \%$ \\
Emergency salvage & $0.2 \%$ & $0.2 \%$ \\
Perfusion time & $92 \%-116)$ & $89(69-113)$ \\
Crossclamp time & $61(45-81)$ & $62(46-82)$ \\
No. of distal anastomoses & & $4.5 \%$ \\
One & $4.7 \%$ & $20.5 \%$ \\
Two & $21.1 \%$ & $74.7 \%$ \\
Three & $73.8 \%$ & \\
IABP timing & & $90.1 \%$ \\
None & $91.1 \%$ & $0.0 \%$ \\
IABP missing timing & $0.2 \%$ & $8.1 \%$ \\
IABP preoperative & $6.7 \%$ & $1.5 \%$ \\
IABP intraoperative & $1.6 \%$ & $0.3 \%$ \\
IABP postoperative & $0.4 \%$ &
\end{tabular}

IABP, Intraaortic balloon pump.
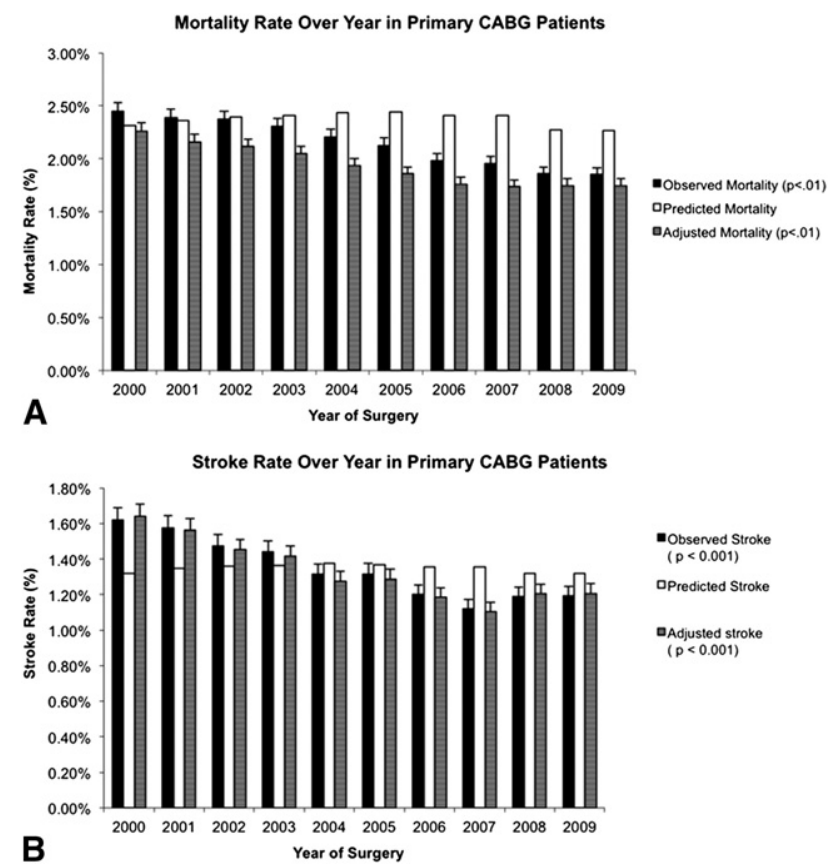

FIGURE 2. Primary outcomes: mortality (A) and stroke (B). Note that stroke definition changed from permanent central nervous system deficit persisting for more than 24 hours during 2000-2008 to a central nervous system deficit persisting for more than 72 hours in 2009. $C A B G$, Coronary artery bypass grafting.

\section{Outcomes in Elective/Urgent Cohort}

Patients undergoing emergency and emergency-salvage operations were removed from the total cohort to evaluate the outcomes after elective CABG. As shown in Table 4, there was a $26.5 \%$ and $27.4 \%$ relative reduction in the observed operative mortality ( $2.1 \%$ vs $1.6 \%)$ and stroke rates $(1.6 \%$ vs $1.1 \%)$ in the combined elective/urgent population, respectively. Similar to findings in the total cohort, there was also a $10.1 \%$ relative reduction in the incidence of reoperation for bleeding $(2.4 \%$ vs $2.1 \%)$ and a $32.7 \%$ relative reduction in the incidence of sternal wound infection $(0.54 \%$ vs $0.36 \%$, Table 4$)$. There was a $26.5 \%$ reduction in the incidence of new-onset renal failure in the combined elective/urgent population. Similar to the findings in the total cohort, there was a $6.7 \%$ relative increase in the incidence of new postoperative atrial fibrillation $(19.7 \%$ vs $21.0 \%)$.

\section{DISCUSSION}

By using the STS Adult Cardiac Surgery Database, this retrospective 10-year study consisting of 1.4 million patients identifies a number of important trends in patient characteristics, operative approaches, and outcomes among patients undergoing CABG. Although patient risk profiles have evolved significantly, the overall predicted risk of mortality remained constant. Despite this overall unchanged 
TABLE 4. Outcomes in total cohort and nonemergency/salvage subcohort

\begin{tabular}{|c|c|c|c|c|c|c|c|c|}
\hline & \multicolumn{4}{|c|}{ Total cohort } & \multicolumn{4}{|c|}{ Nonemergency/salvage sub-cohort } \\
\hline & 2000 & 2009 & $P$ value & Relative risk change & 2000 & 2009 & $P$ value & Relative risk change \\
\hline \multicolumn{9}{|l|}{ Mortality } \\
\hline Observed & $2.4 \%$ & $1.9 \%$ & $<.0001$ & $-24.4 \%(-28.7 \%$ to $-20.1 \%)$ & $2.1 \%$ & $1.6 \%$ & $<.0001$ & $-26.5 \%(-31.2 \%$ to $-21.8 \%)$ \\
\hline Predicted & $2.3 \%$ & $2.3 \%$ & & & $1.9 \%$ & $1.8 \%$ & & \\
\hline Adjusted & $2.3 \%$ & $1.7 \%$ & $<.0001$ & $-22.8 \%(-27.3 \%$ to $-18.3 \%)$ & $2.0 \%$ & $1.5 \%$ & $<.0001$ & $-22.6 \%(-27.6 \%$ to $-17.7 \%)$ \\
\hline \multicolumn{9}{|l|}{ Stroke } \\
\hline Observed & $1.6 \%$ & $1.2 \%$ & $<.0001$ & $-26.4 \%(-31.6 \%$ to $-21.1 \%)$ & $1.6 \%$ & $1.1 \%$ & $<.0001$ & $-27.4 \%(-32.9 \%$ to $-21.9 \%)$ \\
\hline Predicted & $1.3 \%$ & $1.3 \%$ & & & $1.3 \%$ & $1.3 \%$ & & \\
\hline Adjusted & $1.6 \%$ & $1.2 \%$ & $<.0001$ & $-26.4 \%(-31.6 \%$ to $-21.2 \%)$ & $1.6 \%$ & $1.2 \%$ & $<.0001$ & $-26.9 \%(-32.4 \%$ to $-21.5 \%)$ \\
\hline \multicolumn{9}{|l|}{ Renal failure } \\
\hline Observed & $3.5 \%$ & $3.6 \%$ & .005 & $5.5 \%(1.6 \%-9.3 \%)$ & $2.1 \%$ & $1.6 \%$ & $<.0001$ & $-26.5 \%(-31.2 \%$ to $-21.8 \%)$ \\
\hline Predicted & $3.8 \%$ & $4.0 \%$ & & & $1.9 \%$ & $1.8 \%$ & & \\
\hline Adjusted & $3.2 \%$ & $3.2 \%$ & .956 & $-0.1 \%(-4.1 \%$ to $3.9 \%)$ & $2.0 \%$ & $1.5 \%$ & $<.0001$ & $-22.6 \%(-27.6 \%$ to $-17.7 \%)$ \\
\hline \multicolumn{9}{|c|}{ Reoperation for bleed } \\
\hline Observed & $2.5 \%$ & $2.2 \%$ & $<.0001$ & $-9.2 \%(-13.6 \%$ to $-4.7 \%)$ & $2.4 \%$ & $2.1 \%$ & $<.0001$ & $-10.1 \%(-14.8 \%$ to $-5.5 \%)$ \\
\hline Predicted & $2.5 \%$ & $2.4 \%$ & & & $2.4 \%$ & $2.3 \%$ & & \\
\hline Adjusted & $2.4 \%$ & $2.2 \%$ & .002 & $-7.1 \%(-11.6 \%$ to $-2.5 \%)$ & $2.3 \%$ & $2.1 \%$ & $<.0001$ & $-7.3 \%(-12.1 \%$ to $-2.6 \%)$ \\
\hline \multicolumn{9}{|c|}{ Deep sternal wound infection } \\
\hline Observed & $0.55 \%$ & $0.37 \%$ & $<.0001$ & $-32.9 \%(-41.8 \%$ to $-23.9 \%)$ & $0.54 \%$ & $0.36 \%$ & $<.0001$ & $-32.7 \%(-42.0 \%$ to $-23.5 \%)$ \\
\hline Predicted & $0.41 \%$ & $0.43 \%$ & & & $0.40 \%$ & $0.42 \%$ & & \\
\hline Adjusted & $0.59 \%$ & $0.37 \%$ & $<.0001$ & $-37.3 \%(-45.8 \%$ to $-28.7 \%)$ & $0.57 \%$ & $0.36 \%$ & $<.0001$ & $-36.9 \%(-45.8 \%$ to $-28.0 \%)$ \\
\hline \multicolumn{9}{|c|}{ Atrial fibrillation } \\
\hline Observed & $19.8 \%$ & $21.1 \%$ & $<.0001$ & $6.4 \%(4.9 \%-7.8 \%)$ & $19.7 \%$ & $21.0 \%$ & $<.0001$ & $6.7 \%(5.2 \%-8.2 \%)$ \\
\hline Predicted & $20.3 \%$ & $21.2 \%$ & & & $20.2 \%$ & $21.0 \%$ & & \\
\hline Adjusted & $20.0 \%$ & $20.5 \%$ & .004 & $2.2 \%(0.7 \%-3.6 \%)$ & $19.9 \%$ & $20.4 \%$ & .001 & $2.5 \%(1.0 \%-4.0 \%)$ \\
\hline
\end{tabular}

risk, outcomes have substantially improved, with mortality and stroke rates decreasing by $24.4 \%$ and $26.4 \%$, respectively. In addition, there have been significant reductions in the risks of reoperation for bleeding and deep sternal wound infections in the total cohort and non-emergency subcohort, as well as a reduction in postoperative renal failure only in non-emergency patients. Of note, patients undergoing $\mathrm{CABG}$ in the current era are currently receiving superior revascularization conduits and configurations, ${ }^{11,12}$ including more frequent use of the ITA $(94.7 \%$ vs $87.7 \%)$ and nearly all patients receiving at least 1 arterial bypass graft. Although this study is limited in that it does not contain long-term outcomes, the conduits that are currently used are likely to result in superior patency, freedom from angina, and long-term survival compared with patients undergoing CABG 10 years ago. ${ }^{13-16}$

Many studies compare outcomes between CABG and PCI over a medium to long-term time horizon, ${ }^{17-21}$ failing to take into account the incremental improvement in outcomes that occurs as a result of improvements in the inherent safety of surgery. This substantial body of literature comparing outcomes after CABG versus PCI may be somewhat dated, and conclusions from those studies may not be valid in the current era because of the progressive improvement in quality and outcomes of CABG demonstrated here. This study illustrates that there has been a steady and dramatic improvement in outcomes over the past decade that are not due to decreasing patient risk, but rather to improvements in the performance and safety of the surgical procedure and preoperative and postoperative medical treatment. There are a plethora of data indicating that surgery is superior in terms of target vessel revascularization ${ }^{18}$ and freedom from revascularization, ${ }^{22}$ especially in patients with complex lesions and multivessel disease. ${ }^{18}$ Thus, in addition to gaining the long-term revascularization and survival benefits after surgery, patients undergoing $\mathrm{CABG}$ in the current era also benefit from improved decreased short-term complications, such as operative mortality, stroke, sternal wound infections, reoperations for bleeding, and renal failure requiring dialysis, a phenomenon that is just now being identified in the most recent studies comparing $\mathrm{CABG}$ and $\mathrm{PCI}{ }^{22}$

One of the major findings of this study is the use of superior conduits (particularly the left ITA) during the time period analyzed. Although a major factor in the improvement in compliance is likely due to research indicating improved patient survival and patency, ${ }^{11,13-15}$ the STS database has been instrumental in achieving this target by providing surgeons with regular reports of their performance relative to national benchmarks, ultimately facilitating improvement in the quality of care delivered. Further, use of the ITA and mortality are both quality indicators in the STS CABG quality measurement initiative. ${ }^{23,24}$ Additional factors that may be contributing 
to the improvement in results are improved preoperative medical optimization (as demonstrated by improved compliance with medications such as aspirin, statins, beta-blockers, and angiotensin-converting enzyme inhibitors), improved postoperative critical care, and improved performance in the operating room. Although it is beyond the scope of this article to identify the impact of these factors, previous literature has documented significant improvements in each of these areas in the past decade.

The change in the demographics, hemodynamics, and anatomic characteristics reflect the prevalence of PCI and the improved effectiveness of preventative medicine and pharmacologic therapy. During the past decade, there has been a $9.0 \%$ absolute increase in the number of patients presenting with more than $50 \%$ occlusion of the left main coronary artery. Furthermore, patients in the current era are presenting with a high frequency of comorbid conditions likely due to literature that has indicated significant short and longterm benefit in patients with a high atherosclerotic burden and multivessel disease $\mathrm{e}^{25,26}$ in CABG versus PCI.

\section{Limitations}

The strengths of this study are in its large sample size reflecting a large majority of CABGs that have been performed in the United States from 2000 to 2009. As such, these results are robust and generalizable to the entire population of patients undergoing CABG. One key limitation of the STS Adult Cardiac Surgery Database is that it is limited to short-term outcomes. Although there is evidence to suggest that long-term survival may be improved in the cohort of patients currently undergoing CABG given the superiority of conduits, there was no effort to assess these outcomes.

\section{CONCLUSIONS}

Over the past decade there have been significant reductions in the incidences of mortality, stroke, reoperations for bleeding, sternal wound infections, and new-onset renal failure in non-emergency patients. These outcomes seem to reflect improvements in the surgical procedure, rather than a change in the operative risk of patients currently undergoing CABG. Elective CABG is now performed in North America with a mortality rate of $1.6 \%$ and a stroke rate of $1.1 \%$. Although these improvements in outcomes are significant, future challenges for cardiac surgery as a specialty are to focus on continued reduction of mortality of CABG, stroke, and renal failure. These outcomes should serve as benchmarks for competing therapies for multivessel cardiovascular disease.

\section{References}

1. Ford ES, Capewell S. Proportion of the decline in cardiovascular mortality disease due to prevention versus treatment: public health versus clinical care. Annu Rev Public Health. 2011;32:5-22.
2. Ulrich MR, Brock DM, Ziskind AA. Analysis of trends in coronary artery bypass grafting and percutaneous coronary intervention rates in Washington state from 1987 to 2001. Am J Cardiol. 2003;92:836-9.

3. Hlatky MA, Boothroyd DB, Bravata DM, et al. Coronary artery bypass surgery compared with percutaneous coronary interventions for multivessel disease: a collaborative analysis of individual patient data from ten randomised trials. Lancet. 2009;373:1190-7.

4. Serruys PW, Morice M, Kappetein AP, et al. Percutaneous coronary intervention versus coronary-artery bypass grafting for severe coronary artery disease. $\mathrm{NEngl}$ J Med. 2009;360:961-72.

5. Hannan EL, Racz MJ, Walford G, et al. Long-term outcomes of coronary-artery bypass grafting versus stent implantation. N Engl J Med. 2005;352:2174-83.

6. Yusuf S, Zucker D, Peduzzi P, et al. Effect of coronary artery bypass graft surgery on survival: overview of 10 -year results from randomised trials by the Coronary Artery Bypass Graft Surgery Trialists Collaboration. Lancet. 1994; 344:563-70.

7. Taggart DP. Thomas B. Ferguson Lecture. Coronary artery bypass grafting is still the best treatment for multivessel and left main disease, but patients need to know. Ann Thorac Surg. 2006;82:1966-75.

8. Ulrich MR, Brock DM, Ziskind AA. Analysis of trends in coronary artery bypass grafting and percutaneous coronary intervention rates in Washington state from 1987 to 2001. Am J Cardiol. 2003;92:836-9.

9. Riley RF, Don CW, Powell W, Maynard C, Dean LS. Trends in coronary revascularization in the United States from 2001 to 2009: recent declines in percutaneous coronary intervention volumes. Circ Cardiovasc Qual Outcomes. 2011;4: 193-7.

10. Shahian DM, O'Brien SM, Filardo G, et al. The Society of Thoracic Surgeons 2008 cardiac surgery risk models: part 1 - coronary artery bypass grafting surgery. Ann Thorac Surg. 2009;88(1 Suppl):S2-22.

11. Loop FD, Lytle BW, Cosgrove DM, et al. Influence of the internal-mammaryartery graft on 10-year survival and other cardiac events. N Engl J Med. 1986; 314:1-6.

12. Manabe S, Fukui T, Tabata M, et al. Arterial graft deterioration one year after coronary artery bypass grafting. J Thorac Cardiovasc Surg. 2010;140:1306-11.

13. Taggart DP, D'Amico R, Altman DG. Effect of arterial revascularisation on survival: a systematic review of studies comparing bilateral and single internal mammary arteries. Lancet. 2001;358:870-5.

14. Zacharias A, Schwann TA, Riordan CJ, et al. Late results of conventional versus all-arterial revascularization based on internal thoracic and radial artery grafting. Ann Thorac Surg. 2009;87:19-26 e2.

15. Lytle BW, Loop FD, Cosgrove DM, et al. Long-term (5 to 12 years) serial studies of internal mammary artery and saphenous vein coronary bypass grafts. $J$ Thorac Cardiovasc Surg. 1985;89:248-58.

16. Cameron A, Davis KB, Green G, Schaff HV. Coronary bypass surgery with internal-thoracic-artery grafts-effects on survival over a 15-year period. N Engl J Med. 1996;334:216-9.

17. Kim YH, Park DW, Kim WJ, et al. Impact of the extent of coronary artery disease on outcomes after revascularization for unprotected left main coronary artery stenosis. J Am Coll Cardiol. 2010;55:2544-52.

18. Park DW, Kim YH, Yun SC, et al. Long-term outcomes after stenting versus coronary artery bypass grafting for unprotected left main coronary artery disease: 10-year results of bare-metal stents and 5-year results of drug-eluting stents from the ASAN-MAIN (ASAN Medical Center-Left MAIN Revascularization) Registry. J Am Coll Cardiol. 2010;56:1366-75.

19. Hueb W, Lopes NH, Gersh BJ, et al. Five-year follow-up of the Medicine, Angioplasty, or Surgery Study (MASS II): a randomized controlled clinical trial of 3 therapeutic strategies for multivessel coronary artery disease. Circulation. 2007;115:1082-9.

20. Rodriguez A, Bernardi V, Navia J, et al. Argentine randomized study: Coronary Angioplasty with Stenting versus Coronary Bypass Surgery in patients with Multiple-Vessel Disease (ERACI II): 30-day and one-year follow-up results. ERACI II Investigators. J Am Coll Cardiol. 2001;37:51-8.

21. Serruys PW, Unger F, Sousa JE, et al. Comparison of coronary-artery bypass surgery and stenting for the treatment of multivessel disease. N Engl J Med. 2001; 344:1117-24.

22. Hannan EL, Wu C, Walford G, et al. Drug-eluting stents vs coronary-artery bypass grafting in multivessel coronary disease. $N$ Engl J Med. 2008;358: $331-41$.

23. Shahian DM, Edwards FH, Ferraris VA, et al. Quality measurement in adult cardiac surgery: part 1-Conceptual framework and measure selection. Ann Thorac Surg. 2007;83(4 Suppl):S3-12. 
24. O'Brien SM, Shahian DM, DeLong ER, et al. Quality measurement in adult cardiac surgery: part 2-Statistical considerations in composite measure scoring and provider rating. Ann Thorac Surg. 2007;83(4 Suppl):S13-26.

25. Comparison of coronary bypass surgery with angioplasty in patients with multivessel disease. The Bypass Angioplasty Revascularization Investigation (BARI) Investigators. N Engl J Med. 1996;335:217-25.

26. Sculpher MJ, Seed P, Henderson RA, et al. Health service costs of coronary angioplasty and coronary artery bypass surgery: the Randomised Intervention Treatment of Angina (RITA) trial. Lancet. 1994;344:927-30.

\section{Discussion}

Dr T. Bruce Ferguson (Greenville, NC). This nice analysis of major patient risk factor observational processes and outcomes compared between 2000 and 2009 (>1.4 million patients) is an excellent update. They did a nice job of handling different versions of the STS database data in their trends over time analysis. The study clearly documented continued improvement in CABG outcomes, particularly in the CABG PCI era of the last decade.

I have 2 questions for the authors. The first is, you appropriately attribute improvement to better perioperative management overall, but 25\% of the patients in 2009 had prior PCI and 32\% had left main disease, and the number of distal anastomoses increased from 2000 to 2009 . These are clearly more technically difficult operations. Given your study and the recent JAMA report on the overall decline in CABG volumes during this same time frame, although the number of CABG sites has increased, do you foresee a reversal in this trend as sites perform fewer and fewer CABG procedures, and if so, what might be done about this at the local level?

The second question is that you discussed CABG versus PCI outcomes in your article and the difference in the SYNTAX mortality outcomes. If you substitute the SYNTAX PCI arm data with the fractional flow reserve-guided PCI data from FFR versus Angiography for Guiding PCI in Patients with Multivessel Coronary Artery Disease (FAME), the mortality benefit from CABG disappears. While this is being tested directly in the National Heart, Lung, and Blood Institute ischemia trial, which is just getting under way, what do you recommend as options to further improve CABG outcomes in the future to keep up with our interventional colleagues?

Dr ElBardissi. With regard to your first question, a number of studies recently have shown that although there was a significant decline in number of CABGs performed immediately after PCI was adopted and certainly through the 1980s and 1990s, there has certainly been a more recent decrease in the decline in CABG volume. Furthermore, we believe that with studies like these showing excellent outcomes after CABG, we continue to expect, at the very least, the decline in the number of CABGs to temper even further.

Dr Ferguson. Sorry. My question was the decline in the improvement in outcomes from CABG, not the decline in the number of CABGs that are done.

Dr ElBardissi. That is a very different question. It certainly is possible that average institutional volumes will decline as a result of the combination of decreasing global CABG volumes and an increase in the number of hospitals performing $\mathrm{CABG}$, and lower volumes could reverse the trend toward improved outcomes. The volume-outcome relationship for CABG is not as strong as for some other cardiac operations. It should be noted that these data include only immediate postoperative outcomes, thus making it hard to infer potential differences in long-term outcomes, including long-term survival and revascularization.

I think your second question referred to CABG versus PCI outcomes and recommending additional interventions or therapies that would potentially improve the performance of CABG in relative to PCI. This study clearly illustrates we are performing CABG on a very different cohort of patients and patients compared with 10 years ago.

It is essential that we continue to focus on reducing not only mortality but also 2 key morbidities: stroke and renal failure. There remains room for improvement here.

Dr Paul Kurlansky (Miami, Fla). A very interesting study. It parallels some work that was done by Dr Ferguson and colleagues with regard to the STS database in the 1990s, who showed an increasing risk and declining mortality for CABG surgery, and similar findings have been shown in the New York State database over time with increasing risk and declining mortality. I was wondering if you would care to comment on the potential impact of large registries, which make us all aware of our results, on the actual outcomes that we have been observing?

Dr ElBardissi. This is by far the largest cohort of patients we have reviewed (1.4 million patients over the past decade), and we have done a rigorous analysis to not only adjust for preoperative risk but also ensure that the variables we are looking at are consistent over time. I think all of us in this room recognize that the STS database is one of the most valuable assets of the specialty of cardiac surgery.

The major implication of this study is that we are in fact operating on a more complex cohort of patients and doing a better job at getting them through the immediate postoperative phase with less complications and particularly less mortality and less stroke than they were not too long ago, 10 years ago.

The STS database was set up for quality improvement, and the results of this study suggest that it has succeeded in that role. By providing cardiac surgeons with regular reports that include riskadjusted outcomes for CABG and other cardiac operations along with national and regional benchmarks, the STS database has catalyzed improvement in both process and outcomes. The process of measuring and reporting outcomes has been a powerful stimulus for practice improvement.

Dr James Tatoulis (Melbourne, Australia). I noticed that less than $5 \%$ of patients were having bilateral ITAs despite the cohort having a median age of 65 years. Could you comment on how this has changed in the last 10 years, particularly now the population is aging and patients live longer, and whether you can use this type of study to perhaps stimulate some progress in that area?

Dr EIBardissi. Bilateral ITAs at the beginning of our study were $3.5 \%$, and it just increased slightly to $4.1 \%$ at the end of the study; an increase in the use of bilateral ITAs but certainly not a large increase. Certainly increased focus on the rate of bilateral ITA grafting might lead to increased adoption of this strategy.

Dr Ottavio Alfieri (Milan, Italy). What is your personal interpretation of the improvements? If you could identify just a single factor affecting this improvement, what do you think it is?

Dr ElBardissi. We have seen improvements on many fronts. We have seen improvements on process-oriented fronts, surgical decision-making fronts, such as use of the ITA, and then we 
have also seen improvements on the outcome fronts, mortality, stroke rate, and so forth. The process-orientated fronts and surgical decision-making fronts tend to reflect, again, the rigorous clinical studies that are ongoing in the field of cardiac surgery that dictate to us as cardiac surgeons what the optimal revascularization configuration and strategies are going forward in any particular group of patients.

The changes in outcomes we have seen, again, adjusting for patient risk and still seeing a significant decline in mortality and stroke rate, reflect a number of things. Number one, they reflect more optimal medical management when patients come into surgery. Something that shouldn't be lost is that this is not just better care being received in the operating room, this is probably better perioperative surgical care, preoperative, operative, and intensive care unit level care and floor level care. So I think there are a number of things are going on here. It is difficult to know from our study exactly what is contributing to the improved outcomes we have seen over the past decade, but certainly better intraoperative decision-making and better perioperative surgical care. 